\title{
Analysis of the $B^{+} \rightarrow J / \psi \phi K^{+}$data at low $J / \psi \phi$ invariant masses and the $X(4140)$ and $X(4160)$ resonances
}

\author{
En Wang, ${ }^{1}$ Ju-Jun Xie, ${ }^{2}$ Li-Sheng Geng, ${ }^{3}$ and Eulogio Oset ${ }^{2,4}$ \\ ${ }^{1}$ Department of Physics, Zhengzhou University, Zhengzhou, Henan 450001, China \\ ${ }^{2}$ Institute of Modern Physics, Chinese Academy of Sciences, Lanzhou 730000, China \\ ${ }^{3}$ School of Physics and Nuclear Energy Engineering and International Research Center for Nuclei \\ and Particles in the Cosmos, Beihang University, Beijing 100191, China \\ ${ }^{4}$ Departamento de Física Teórica and IFIC, Centro Mixto Universidad de Valencia-CSIC \\ Institutos de Investigación de Paterna, Aptdo. 22085, 46071 Valencia, Spain
}

(Received 11 October 2017; published 25 January 2018)

\begin{abstract}
We have studied the $J / \psi \phi$ mass distribution of the $B^{+} \rightarrow J / \psi \phi K^{+}$reaction from threshold to about $4250 \mathrm{MeV}$, and find that one needs the contribution of the $X(4140)$ with a narrow width, together with the $X(4160)$ which accounts for most of the strength of the distribution in that region. The existence of a clear cusp at the $D_{s}^{*} \bar{D}_{s}^{*}$ threshold indicates that the $X(4160)$ resonance is strongly tied to the $D_{s}^{*} \bar{D}_{s}^{*}$ channel, which finds a natural interpretation in the molecular picture of this resonance.
\end{abstract}

DOI: $10.1103 /$ PhysRevD.97.014017

The recent measurement of the $B^{+} \rightarrow J / \psi \phi K^{+}$reaction at $\mathrm{LHCb}[1,2]$ and analysis of the data has brought some surprises as the claim of several new states that couple to $J / \psi \phi$. Another surprise is that the $X(4140)$ deduced from the analysis, with quantum numbers $J^{P C}=1^{++}$, however has a width $\Gamma \approx 83 \pm 24_{-14}^{+21} \mathrm{MeV}$, substantially larger than that claimed in the former experiments $\mathrm{CDF}[3,4], \mathrm{LHCb}$ [5], CMS [6], D0 [7,8], and the average $19_{-7}^{+8} \mathrm{MeV}$ of the PDG [9].

In this work, we show that the low invariant $J / \psi \phi$ mass region, manifestly improvable in the fit of Refs. [1,2], requires the contributions of a narrow $X(4140)$ and a wide $X(4160)$ resonance which couples to $J / \psi \phi$ but is mostly made by a $D_{s}^{*} \bar{D}_{s}^{*}$ molecule. As a consequence of analyticity and driven by the molecular nature of $X(4160)$, the $J / \psi \phi$ mass spectrum develops a strong cusp around the $D_{s}^{*} \bar{D}_{s}^{*}$ threshold that the fit of Refs. [1,2] cannot reproduce because this mechanism is not contemplated in the amplitude that is fitted to the data.

The $X(4140)$ has been the subject of much theoretical work and multiple suggestions have been given about its possible structure $[10,11]$. Here we briefly discuss the works where claims of a $D_{s}^{*} \bar{D}_{s}^{*}$ molecule have been made. Effective Lagrangians accounting for pseudoscalar and vector exchange are used in Ref. [12], together with triangle

Published by the American Physical Society under the terms of the Creative Commons Attribution 4.0 International license. Further distribution of this work must maintain attribution to the author(s) and the published article's title, journal citation, and DOI. Funded by SCOAP. diagrams to connect $D_{s}^{*} \bar{D}_{s}^{*}$ to $J / \psi \phi$. A state that could be associated with $X(4140)$ was obtained with $J^{P C}=0^{++}$or $2^{++}$. In Ref. [13], a different strategy is followed involving the Weinberg compositeness condition $[14,15]$ and getting the couplings from the binding energy of $D_{s}^{*} \bar{D}_{s}^{*}$. Once again $0^{++}$or $2^{++}$are the preferred quantum numbers. In Ref. [16], vector meson exchange, together with the Bethe Salpeter equations are used and once more a $0^{++}$structure is favored. The possibility that the $X(4140)$ is a $D_{s}^{*} \bar{D}_{s}^{*}$ molecule is also discussed in Ref. [17], where $\eta$ exchange is used to connect heavy mesons which have no $u, d$ quarks, although the authors admit that other ingredients apart from $\eta$ exchange would be needed to bind that state. QCD sum rules have also contributed to this discussion and in Refs. [18-21], although with uncertainties of about $100 \mathrm{MeV}$ in the mass, the possibility that the $X(4140)$ corresponds to a $D_{s}^{*} \bar{D}_{s}^{*}$ molecule with $0^{++}$is pointed out. A study of this state using $\eta, \phi$, and $\sigma$ exchange is done in Ref. [22], concluding that the $X(4140)$ could correspond to a $D_{s}^{*} \bar{D}_{s}^{*}$ molecule with $0^{++}$although $0^{-+}$or $2^{++}$were not excluded.

A thorough study of $X$ states emerging from the interaction of vector pairs, light-light (with light $\equiv$ $\rho, \omega, \phi, K^{*}$ ), light-heavy (with heavy $\equiv D^{*}, \quad D_{s}^{*}$ and $J / \psi$ ), and heavy-heavy, was done in Ref. [23] and several states were obtained that could be associated to known states. In particular, as relevant for the present work, a state $0^{+}\left(2^{++}\right)$at $4169 \mathrm{MeV}$ was obtained, coupling mostly to $D_{s}^{*} \bar{D}_{s}^{*}$, that qualifies as a $D_{s}^{*} \bar{D}_{s}^{*}$ molecule and was associated to the $X(4160)$, not the $X(4140)$. It is interesting to see that the same conclusions are reached by using QCD sum rules in Ref. [24]. The dynamics used in Ref. [23] is 
based on the local hidden gauge approach [25-27], exchanging vector mesons and including contact terms. The channels included in the interaction are $D^{*} \bar{D}^{*}, D_{s}^{*} \bar{D}_{s}^{*}$, $K^{*} \bar{K}^{*}, \rho \rho, \omega \omega, \phi \phi, J / \psi J / \psi, \omega J / \psi, \phi J / \psi, \omega \phi$. It was shown there that the couplings of the state to the $D_{s}^{*} \bar{D}_{s}^{*}$ was dominant $\left(g_{D_{s}^{*} \bar{D}_{s}^{*}}=18927-5524 i \mathrm{MeV}\right)$, followed by the one to $\phi J / \psi\left(g_{J / \psi \phi}=-2617-5151 i \mathrm{MeV}\right)$. The coupling to $D^{*} \bar{D}^{*}$ is $g_{D^{*} \bar{D}^{*}}=1225-490 i$, sizeable enough, which guarantees that this resonance can be seen in the $D^{*} \bar{D}^{*}$ channel. Actually, this is the channel where the $X(4160)$ was observed in the $e^{+} e^{-} \rightarrow J / \psi X, X \rightarrow D^{*} \bar{D}^{*}$ reaction [28]. The width of the $X(4160)$ is given by $\Gamma=139_{-61}^{+119} \pm 21 \mathrm{MeV}$, much wider than that of the $X(4140)$. The work of Ref. [23] gives $\Gamma=132 \pm$ $25 \mathrm{MeV}$. It should be noticed that with the coupling $g_{J / \psi \phi}$ found, one obtains a partial decay width $\Gamma_{J / \psi \phi} \approx$ $22 \mathrm{MeV}$. Hence, much of the width comes from other channels, in particular the light-light ones that have much phase space for the decay.

It is interesting to note in retrospect that the theoretical works discussed above that associated the $D_{s}^{*} \bar{D}_{s}^{*}$ structure to the $X(4140)$ could equally have associated it to the $X(4160)$. One can guess that the fact that light-light channels were not considered rendered the width of the state small and the association to the $X(4140)$ state was more natural. Yet, today, with the quantum numbers of the $X(4140)$ established to be $0^{+}\left(1^{++}\right)[1,2,9]$, the association of the $0^{++}, 2^{++}$states to the $X(4140)$ can no longer be supported, but the idea of the $D_{s}^{*} \bar{D}_{s}^{*}$ molecule associated to the $X(4160)$ has much weight.

The dominant terms of the interaction in Ref. [23] correspond to the exchange of light vectors, and they can be obtained from the picture where the heavy quarks of the vector components are spectators, and only light quarks are operative in the interaction $[29,30]$. This allows us to obtain this interaction from the SU(3) light sector, concretely from the $\bar{K} N \rightarrow \bar{K} N$ amplitude where the $s$ quark of the $\bar{K}$ is also a spectator and $\phi$ is not exchanged, and at the same time, it guarantees that heavy quark symmetry is fulfilled since the heavy quarks are spectators in the interaction. Transitions from heavy-heavy to light-light require the exchange of heavy vectors leading to subdominant terms in the inverse of the heavy quark mass counting, which are calculated using SU(4) symmetry and one accepts as being model dependent. From this perspective, we will allow the total width of the $X(4160)$ coming from the light-light channels to be somewhat different than the one obtained in Ref. [23] when we fit the data of Refs. [1,2].

Next we proceed to apply our approach to the data of Refs. [1,2] which we consider from threshold up to about $4250 \mathrm{MeV}$, above the $D_{s}^{*} \bar{D}_{s}^{*}$ threshold. The data show a narrow peak around $4140 \mathrm{MeV}$, followed by one broader structure around $4160-4170 \mathrm{MeV}$, and a remarkable cusp structure around the $D_{s}^{*} \bar{D}_{s}^{*}$ threshold. The presence of a cusp at the $D_{s}^{*} \bar{D}_{s}^{*}$ threshold in the $J / \psi \phi$ mass distribution clearly indicates a link of the resonance responsible for the $J / \psi \phi$ spectrum with the $D_{s}^{*} \bar{D}_{s}^{*}$ channel. This link can be provided assuming that the $X(4160)$ is mostly responsible for this spectrum.

The next step requires us to discuss how a $D_{s}^{*} \bar{D}_{s}^{*}$ resonance is produced in the weak decay $B^{-} \rightarrow K^{-} D_{s}^{*} \bar{D}_{s}^{*}$ (we take the complex conjugate reaction to deal with $b$ quark rather than $\bar{b}$ quark). The dominant process at the quark level proceeds as shown in Fig. 1, involving external emission [31]. This allows us immediately to obtain the $B^{-} \rightarrow K^{-} D_{s}^{*} \bar{D}_{s}^{*}$ amplitude in the region around the $X(4160)$ resonance as depicted in Fig. 2. Obviously in the neighborhood of the resonance the tree level term of Fig. 2(a) is small compared to the resonant term, but we keep it in the calculations. For the production of $J / \psi \phi$ with this mechanism, the tree level of Fig. 2(a) does not contribute and then we are led to the diagram of Fig. 3.

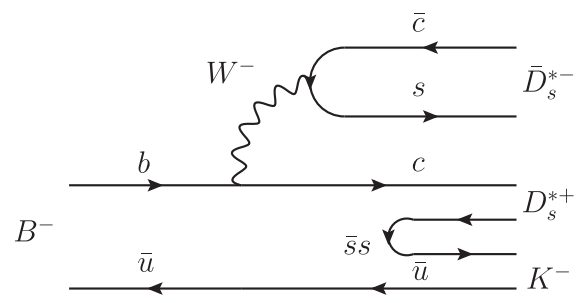

FIG. 1. Microscopic quark level production of $K^{-} D_{s}^{*} \bar{D}_{s}^{*}$ in $B^{-}$ decay.

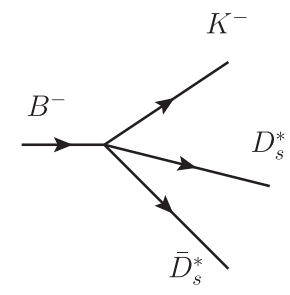

(a)

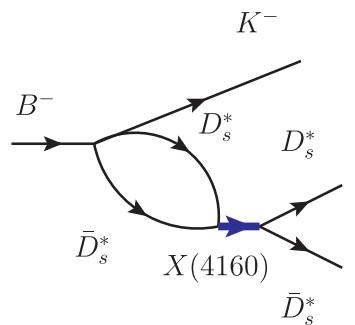

(b)
FIG. 2. Mechanism for $B^{-} \rightarrow K^{-} D_{s}^{*} \bar{D}_{s}^{*}$ in the presence of the $X(4160)$ resonance.

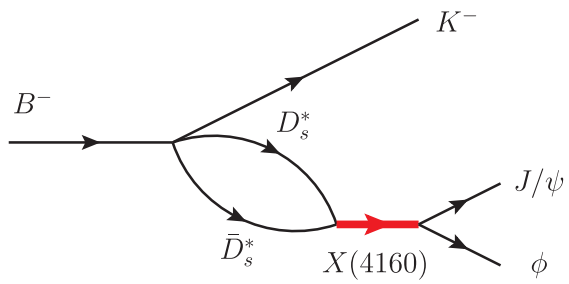

FIG. 3. Mechanism for $B^{-} \rightarrow K^{-} J / \psi \phi$ driven by the $X(4160)$ resonance. 


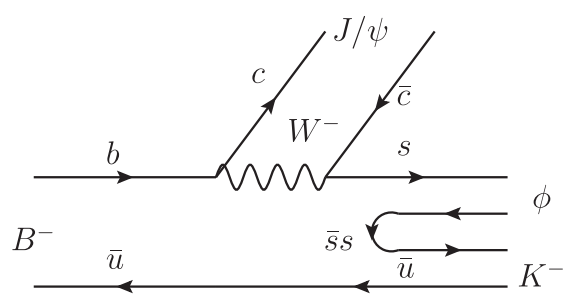

FIG. 4. Tree level diagram to produce the $B^{-} \rightarrow K^{-} J / \psi \phi$ decay involving internal conversion.

The $B^{-} \rightarrow K^{-} J / \psi \phi$ reaction can also proceed through the mechanism of Fig. 4 involving internal conversion. Yet, the internal conversion is penalized by color factors with respect to the external emission of Fig. 1, and hence this term, or rescattering of this term like that in Fig. 3, but with $J / \psi \phi$ intermediate state instead of $D_{s}^{*} \bar{D}_{s}^{*}$, which would involve the extra factor $g_{J / \psi \phi} / g_{D_{s}^{*} \bar{D}_{s}^{*}}$ versus the amplitude of Fig. 3, can be safely neglected.

We can then write the amplitudes for the $B^{-} \rightarrow K^{-} J / \psi \phi$ and $B^{-} \rightarrow K^{-} D_{s}^{*} \bar{D}_{s}^{*}$. The resonance $X(4160)$ obtained in Ref. [23] is a $J^{P C}=2^{++}$state with $L=0$ in $D_{s}^{*} \bar{D}_{s}^{*}$. To match angular momentum in the reaction, we need a $D$-wave in the $K^{-}$and the amplitude is thus of the type,

$$
t_{B^{-} \rightarrow K^{-} D_{s}^{*} \bar{D}_{s}^{*}}^{\text {tree }}=A\left(\vec{\epsilon} \cdot \vec{k} \vec{\epsilon}^{\prime} \cdot \vec{k}-\frac{1}{3} \vec{k}^{2} \vec{\epsilon} \cdot \vec{\epsilon}^{\prime}\right)
$$

where $\vec{\epsilon}, \vec{\epsilon}^{\prime}$ are the polarization vectors of $D_{s}^{*}$ and $\vec{D}_{s}^{*}$, and we evaluate it in the frame of reference where the $D_{s}^{*} \bar{D}_{s}^{*}$ system is at rest. $A$ in Eq. (1) is an unknown factor that will be fitted to the data. The sum over polarizations of $|t|^{2}$ is

$$
\sum_{\text {pol }}\left|t_{B^{-} \rightarrow K^{-} D_{s}^{*} \bar{D}_{s}^{*}}^{\text {tree }}\right|^{2}=\frac{2}{3}|\vec{k}|^{4}
$$

and then for the tree level term of Fig. 2(a) we obtain

$$
\frac{d \Gamma}{d M_{\text {inv }}\left(D_{s}^{*} \bar{D}_{s}^{*}\right)}=\frac{1}{(2 \pi)^{3}} \frac{1}{4 M_{B^{-}}^{2}} \frac{2}{3}|\vec{k}|^{4}\left|\vec{k}^{\prime}\right| \tilde{p}_{D_{s}^{*}}|A|^{2},
$$

where $\vec{k}$, as mentioned before, is the $K^{-}$momentum in the $D_{s}^{*} \bar{D}_{s}^{*}$ rest frame, $\vec{k}^{\prime}$ the $K^{-}$momentum in the $B^{-}$rest frame, and $\tilde{p}_{D_{s}^{*}}$ the $D_{s}^{*}$ momentum in the $D_{s}^{*} \bar{D}_{s}^{*}$ rest frame.

If we want to get the mass distribution for the mechanisms depicted in Fig. 2 for $D_{s}^{*} \bar{D}_{s}^{*}$ production including the $X(4160)$ resonance, we make the following replacement:

$$
\begin{aligned}
A \rightarrow & A\left[1+G_{D_{s}^{*} \bar{D}_{s}^{*}}\left(M_{\mathrm{inv}}\left(D_{s}^{*} \bar{D}_{s}^{*}\right)\right)\right. \\
& \left.\times t_{D_{s}^{*} \bar{D}_{s}^{*} \rightarrow D_{s}^{*} \bar{D}_{s}^{*}}\left(M_{\mathrm{inv}}\left(D_{s}^{*} \bar{D}_{s}^{*}\right)\right)\right] .
\end{aligned}
$$

To obtain the mass distribution for $J / \psi \phi$ through the mechanism of Fig. 3, we make the following replacement in Eq. (3):

$$
\begin{aligned}
A \rightarrow & A \times G_{D_{s}^{*} \bar{D}_{s}^{*}}\left(M_{\mathrm{inv}}(J / \psi \phi)\right) \\
& \times t_{D_{s}^{*} \bar{D}_{s}^{*} \rightarrow J / \psi \phi}\left(M_{\mathrm{inv}}(J / \psi \phi)\right),
\end{aligned}
$$

and $k, k^{\prime}$ are the same $K^{-}$momenta as before suited to the situation of having $J / \psi \phi$ in the final state rather than $D_{s}^{*} \bar{D}_{s}^{*}$. Similarly, $\tilde{p}_{D_{s}^{*}}$ in Eq. (3) is replaced by $\tilde{p}_{\phi}$, which is the $\phi$ momentum in the $J / \psi \phi$ rest frame.

The $G$ function appearing in Eqs. (4) and (5) is the loop function for two intermediate $D_{s}^{*} \bar{D}_{s}^{*}$. To avoid potential dangers using the dimensional regularization as pointed out in Ref. [32], we use the cutoff method with $q_{\max }$ fixed such as to give the same value as $G$ with dimensional regularization used in Ref. [23] at the pole position.

The amplitudes appearing in Eqs. (4) and (5) are given in terms of the $g_{D_{s}^{*} \bar{D}_{s}^{*}}$ and $g_{J / \psi \phi}$ obtained in Ref. [23] by

$$
\begin{aligned}
& t_{D_{s}^{*} \bar{D}_{s}^{*} \rightarrow D_{s}^{*} \bar{s}_{s}^{*}}=\frac{g_{D_{s}^{*} \bar{D}_{s}^{*}}^{2}}{M_{\mathrm{inv}}^{2}\left(D_{s}^{*} \bar{D}_{s}^{*}\right)-M_{X}^{2}+i M_{X} \Gamma_{X}}, \\
& t_{D_{s}^{*} \bar{D}_{s}^{*} \rightarrow J / \psi \phi}=\frac{g_{D_{s}^{*} \bar{D}_{s}^{*}} g_{J / \psi \phi}}{M_{\mathrm{inv}}^{2}(J / \psi \phi)-M_{X}^{2}+i M_{X} \Gamma_{X}},
\end{aligned}
$$

where

$$
\Gamma_{X}=\Gamma_{0}+\Gamma_{J / \psi \phi}+\Gamma_{D_{s}^{*} \bar{D}_{s}^{*}},
$$

with $\Gamma_{0}$ accounting for the channels of Ref. [23] not explicitly considered here (we shall fit that to the data as discussed above), and

$$
\begin{gathered}
\Gamma_{J / \psi \phi}=\frac{\left|g_{J / \psi \phi}\right|^{2}}{8 \pi M_{X}^{2}} \tilde{p}_{\phi}, \\
\Gamma_{D_{s}^{*} \bar{D}_{s}^{*}}=\frac{\left|g_{D_{s}^{*} \bar{D}_{s}^{*}}\right|^{2}}{8 \pi M_{X}^{2}} \tilde{p}_{D_{s}^{*}} \Theta\left(M_{\mathrm{inv}}\left(D_{s}^{*} \bar{D}_{s}^{*}\right)-2 M_{D_{s}^{*}}\right) .
\end{gathered}
$$

Equations (6) and (7) together with Eqs. (9) and (10) incorporate the Flatté effect for the opening of the important $D_{s}^{*} \bar{D}_{s}^{*}$ channel above the $D_{s}^{*} \bar{D}_{s}^{*}$ threshold.

To account for the production of $J / \psi \phi$ via the $1^{++} X(4140)$ resonance, we take the suitable operator with the kaon in $P$-wave $\left(\vec{\epsilon}_{J / \psi} \times \vec{\epsilon}_{\phi}\right) \cdot \vec{k}$, and the mass distribution coming from this source is given by Eq. (3) with the following substitution:

$$
\begin{gathered}
M_{\text {inv }}\left(D_{s}^{*} \bar{D}_{s}^{*}\right) \rightarrow M_{\text {inv }}(J / \psi \phi), \\
\frac{2}{3}|\vec{k}|^{4} \rightarrow 2|\vec{k}|^{2}, \quad \tilde{p}_{D_{s}^{*}} \rightarrow \tilde{p}_{\phi}, \\
A M_{X(4140)}^{4} \\
A \rightarrow \frac{M_{\text {inv }}^{2}(J / \psi \phi)-M_{X(4140)}^{2}+i M_{X(4140)} \Gamma_{X(4140)}}{},
\end{gathered}
$$

with $B$ a parameter to be fitted to the data. Here we take $M_{X(4140)}=4132 \mathrm{MeV}$, since this is the peak of the $X(4140)$ 


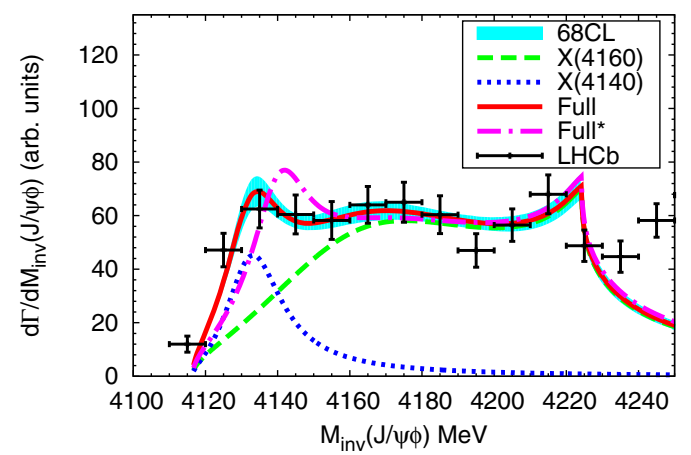

FIG. 5. The $J / \psi \phi$ mass distribution of the $B^{-} \rightarrow K^{-} J / \psi \phi$ decay. The band comes considering the errors in $A, B$ and $\Gamma_{0}$ from the fit, and represents the $68 \%$ confidence level.

structure used in Refs. [1,2], and $\Gamma_{X(4140)}=19 \mathrm{MeV}$. In Refs. [1,2] the authors use both a Breit-Wigner and a structure incorporating the $D_{s} \bar{D}_{s}^{*}$ threshold, as suggested in Ref. [33].

The freedom to fit the data are the parameters $A, B$, and $\Gamma_{0}$. A suitable fit to the data is obtained as shown in Fig. 5 with $\Gamma_{0}=65.0 \pm 7.1 \mathrm{MeV}$ (at $68 \%$ confidence level), which together with $\Gamma_{J / \psi \phi} \simeq 22.0 \mathrm{MeV}$ would provide $\Gamma_{X(4160)} \simeq$ $87.0 \pm 7.1 \mathrm{MeV}$ which is compatible with the experimental width from the PDG of $\Gamma=139_{-61}^{+111} \pm 21 \mathrm{MeV}$. With the fitted parameters, our results (red solid curve, labeled as "Full") are in good agreement with the experimental data at low $J / \psi \phi$ invariant masses, and we also present the uncertainties of our results in Fig. 5. As we can see in the figure, we obtain a contribution from the $X(4140)$ (blue dotted curve) that is dominant at low invariant masses, and is responsible for the peak observed in the experiment around $4135 \mathrm{MeV}$. The $X(4160)$ (green dashed curve) is responsible for most of the strength and produces a broader peak around $4170 \mathrm{MeV}$ (we take the mass $M_{X(4160)}=4169 \mathrm{MeV}$ as obtained in Ref. [23]). And finally a cusp appears at the $D_{s}^{*} \bar{D}_{s}^{*}$ threshold as it shows up in the experiment. This cusp comes from the factor $G_{D_{s}^{*} \bar{D}_{s}^{*}}\left(M_{\text {inv }}\right)$ and reflects the analytical structure of this function with a discontinuity of the derivative at threshold. One must stress that this factor appears here as a consequence of analyticity, with a large strength due to the $D_{s}^{*} \bar{D}_{s}^{*}$ molecular structure of the $X(4160)$. In an analysis like the one of Refs. [1,2], where a sum of amplitudes for resonance excitation and some background are fitted to the data, this factor is not considered, and as a consequence the cusp around $D_{s}^{*} \bar{D}_{s}^{*}$ in the data is missed in the fit. To quantify the improvement in the fit we compute our $\chi^{2}$ up to the datum of $4240 \mathrm{MeV}$, and compare it with the one of Refs. [1,2]. We obtained $\chi^{2}=15.3$ versus $\chi^{2}=22.8$ in Refs. [1,2]. Yet more significative than this is the fact that we obtain the structure of the peaks in good agreement with experiment, while this is not the case in Refs. [1,2].

The Flatté effect is visible in Fig. 5 as a sharp fall down of the invariant mass distribution above the $D_{s}^{*} \bar{D}_{s}^{*}$ threshold.

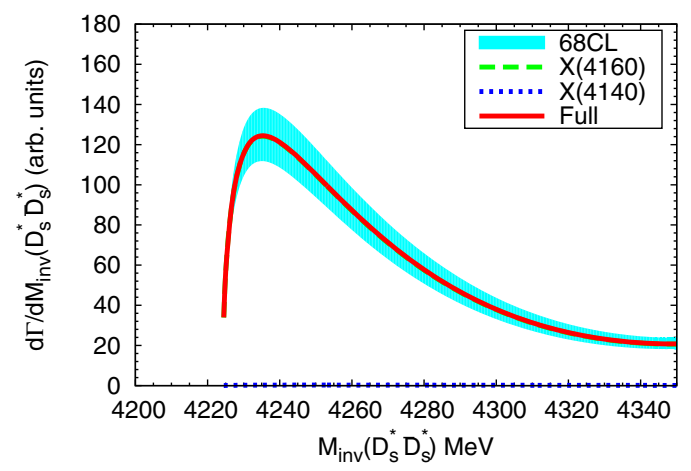

FIG. 6. The $D_{s}^{*} \bar{D}_{s}^{*}$ mass distribution of the $B^{-} \rightarrow K^{-} D_{s}^{*} \bar{D}_{s}^{*}$ decay. The band comes considering the errors in $A, B$ and $\Gamma_{0}$ from the fit, and represents the $68 \%$ confidence level.

From there on, our fit starts diverting from experiment, but so it should, since definitely, other contributions from resonances and backgrounds as discussed in Refs. [1,2], should be considered. Our point is that the lower part of the spectrum can be obtained from the contribution of the $X(4140)\left(1^{++}\right)$and $X(4160)\left(2^{++}\right)$resonances, the $X(4140)$ is narrow, like determined in most experiments, and the cusp of the distribution at the $D_{s}^{*} \bar{D}_{s}^{*}$ threshold indicates that the resonance to which the $J / \psi \phi$ is coupled in that region is strongly tied to the $D_{s}^{*} \bar{D}_{s}^{*}$ channel.

We have conducted the same fit using $M_{X(4140)}=$ $4140 \mathrm{MeV}$ (pink dash-dotted curve in Fig. 5, labeled as "Full*"), and the fit is acceptable but slightly worse in the first several points of the spectrum.

We should note that, some added $2^{++}$structures were not compelling in Refs. [1,2], but once again, our point is that the $2^{++}$state that we add comes in the form of an amplitude $G t$ that cannot be accommodated by a Breit-Wigner amplitude as in Refs. [1,2].

To finish the work, and as a test of the explanation given here, we present in Fig. 6 the $D_{s}^{*} \bar{D}_{s}^{*}$ mass distribution above threshold obtained with the same parameters as in Fig. 5. This should allow a quantitative comparison with the $J / \psi \phi$ mass distribution of Fig. 5, once this experiment is done, which we very much encourage.

As we can see in Fig. 6, there is a peak close to threshold, which should not be misidentified with a new resonance, but it is the reflection of the $X(4160)$ which in our fit has the mass at $4169 \mathrm{MeV}$. The strength at the peak is about twice the one of the $\mathrm{X}(4140)$ in the $J / \psi \phi$ distribution, which guarantees its observability. The $B^{-} \rightarrow K^{-} D_{s}^{*} \bar{D}_{s}^{*}$ mode is not reported in the PDG, but many modes with one $D_{s}^{*}$ already exist. The present work and the prediction, tied to the interpretation given for the $B^{-} \rightarrow K^{-} J / \psi \phi$ spectrum, should act as an incentive to measure this reaction and learn about properties of the $X(4140)$ and $X(4160)$.

Since there are more observables in the experiment, like angular distributions, and other information not available to us, it would also be most convenient that the present ideas and the new algorithm for the analysis are implemented in 
future reanalyses of the $\mathrm{LHCb}$ data. This could also affect other cases. The openness of the LHCb Collaboration to consider these facts [34] opens new windows to further learn about the dynamics of hadrons and their nature.

\section{ACKNOWLEDGMENTS}

We thank T. Skwarnicki and T. Gershon for useful discussions. One of us, E. O., wishes to acknowledge the support from the Chinese Academy of Sciences in the Program of CAS president's International Fellowship for visiting scientists. This work is partly supported by the National Natural Science Foundation of China under Grants No. 11475227, No. 11375024, No. 11522539,
No. 11735003, No. 11505158, No. 11475015, and No. 11647601. It is also supported by the Youth Innovation Promotion Association CAS (No. 2016367), the China Postdoctoral Science Foundation under Grant No. 2015M582197, the Postdoctoral Research Sponsorship in Henan Province under Grant No. 2015023, and Academic Improvement Project of Zhengzhou University. This work is also partly supported by the Spanish Ministerio de Economia y Competitividad and European FEDER funds under Contracts No. FIS2014-57026-REDT, No. FIS2014-51948-C2-1-P, and No. FIS2014-51948-C2-2-P, and the Generalitat Valenciana in the program Prometeo II-2014/068.
[1] R. Aaij et al. (LHCb Collaboration), Amplitude analysis of $B^{+} \rightarrow J / \psi \phi K^{+}$decays, Phys. Rev. D 95, 012002 (2017).

[2] R. Aaij et al. (LHCb Collaboration), Observation of $J / \psi \phi$ Structures Consistent with Exotic States from Amplitude Analysis of $B^{+} \rightarrow J / \psi \phi K^{+}$Decays, Phys. Rev. Lett. 118, 022003 (2017).

[3] T. Aaltonen et al. (CDF Collaboration), Evidence for a Narrow Near-Threshold Structure in the $J / \psi \phi$ Mass Spectrum in $B^{+} \rightarrow J / \psi \phi K^{+}$Decays, Phys. Rev. Lett. 102, 242002 (2009).

[4] T. Aaltonen et al. (CDF Collaboration), Observation of the $Y(4140)$ structure in the $J / \psi \phi$ mass spectrum in $B^{ \pm} \rightarrow$ $J / \psi \phi K^{ \pm}$decays, Mod. Phys. Lett. A 32, 1750139 (2017).

[5] R. Aaij et al. (LHCb Collaboration), Search for the $X(4140)$ state in $B^{+} \rightarrow J / \psi \phi K^{+}$decays, Phys. Rev. D 85, 091103 (2012).

[6] S. Chatrchyan et al. (CMS Collaboration), Observation of a peaking structure in the $J / \psi \phi$ mass spectrum from $B^{ \pm} \rightarrow$ $J / \psi \phi K^{ \pm}$decays, Phys. Lett. B 734, 261 (2014).

[7] V. M. Abazov et al. (D0 Collaboration), Search for the $X(4140)$ state in $B^{+} \rightarrow J / \psi \phi K^{+}$decays with the D0 Detector, Phys. Rev. D 89, 012004 (2014).

[8] V. M. Abazov et al. (D0 Collaboration), Inclusive Production of the $X(4140)$ State in $p \bar{p}$ Collisions at D0, Phys. Rev. Lett. 115, 232001 (2015).

[9] C. Patrignani et al. (Particle Data Group), Review of particle physics, Chin. Phys. C 40, 100001 (2016).

[10] H. X. Chen, W. Chen, X. Liu, and S. L. Zhu, The hiddencharm pentaquark and tetraquark states, Phys. Rep. 639, 1 (2016).

[11] F. K. Guo, C. Hanhart, U. G. Meißner, Q. Wang, Q. Zhao, and B. S. Zou, Hadronic molecules, arXiv:1705.00141.

[12] X. Liu and S. L. Zhu, $Y(4143)$ is probably a molecular partner of $Y(3930)$, Phys. Rev. D 80, 017502 (2009); Erratum: $Y(4143)$ is probably a molecular partner of $Y$ (3930), Phys. Rev. D 85, 019902(E) (2012).

[13] T. Branz, T. Gutsche, and V. E. Lyubovitskij, Hadronic molecule structure of the $Y(3940)$ and $Y(4140)$, Phys. Rev. D 80, 054019 (2009).
[14] S. Weinberg, Evidence that the deuteron is not an elementary particle, Phys. Rev. 137, B672 (1965).

[15] V. Baru, J. Haidenbauer, C. Hanhart, Y. Kalashnikova, and A.E. Kudryavtsev, Evidence that the $a_{0}(980)$ and $f_{0}(980)$ are not elementary particles, Phys. Lett. B 586, 53 (2004).

[16] X. Chen, X. Lü, R. Shi, and X. Guo, Mass of $Y(4140)$ in Bethe-Salpeter equation for quarks, arXiv:1512.06483.

[17] M. Karliner and J. L. Rosner, Exotic resonances due to $\eta$ exchange, Nucl. Phys. A954, 365 (2016).

[18] R. M. Albuquerque, M. E. Bracco, and M. Nielsen, A QCD sum rule calculation for the $Y(4140)$ narrow structure, Phys. Lett. B 678, 186 (2009).

[19] J. R. Zhang and M. Q. Huang, $(Q \bar{s})^{(*)}(\bar{Q} s)^{(*)}$ molecular states from QCD sum rules: A view on $Y(4140)$, J. Phys. G 37, 025005 (2010).

[20] Z. G. Wang, Analysis of the $Y(4140)$ with QCD sum rules, Eur. Phys. J. C 63, 115 (2009).

[21] Z. G. Wang and Y.f. Tian, Tetraquark state candidates: $Y(4140), Y(4274)$ and $X(4350)$, Int. J. Mod. Phys. A 30, 1550004 (2015).

[22] G. J. Ding, Possible molecular states of $D_{s}^{*} \bar{D}_{s}^{*}$ system and Y(4140), Eur. Phys. J. C 64, 297 (2009).

[23] R. Molina and E. Oset, $Y(3940), Z(3930)$, and the $X(4160)$ as dynamically generated resonances from the vector-vector interaction, Phys. Rev. D 80, 114013 (2009).

[24] A. Martnez Torres, K. P. Khemchandani, J. M. Dias, F. S. Navarra, and M. Nielsen, Understanding close-lying exotic charmonia states within QCD sum rules, Nucl. Phys. A966, 135 (2017).

[25] M. Bando, T. Kugo, S. Uehara, K. Yamawaki, and T. Yanagida, Is the $\rho$ Meson a Dynamical Gauge Boson of Hidden Local Symmetry?, Phys. Rev. Lett. 54, 1215 (1985).

[26] M. Bando, T. Kugo, and K. Yamawaki, Nonlinear realization and hidden local symmetries, Phys. Rep. 164, 217 (1988).

[27] U. G. Meißner, Low-energy hadron physics from effective chiral Lagrangians with vector mesons, Phys. Rep. 161, 213 (1988). 
[28] P. Pakhlov et al. (Belle Collaboration), Production of New Charmoniumlike States in $e^{+} e^{-} \rightarrow J / \psi D^{*} \bar{D}^{*}$ at $\sqrt{s} \sim 10 \mathrm{GeV}$, Phys. Rev. Lett. 100, 202001 (2008).

[29] S. Sakai, L. Roca, and E. Oset, Charm-beauty meson bound states from $B\left(B^{*}\right) D\left(D^{*}\right)$ and $B\left(B^{*}\right) \bar{D}\left(\bar{D}^{*}\right)$ interaction, Phys. Rev. D 96, 054023 (2017).

[30] V. R. Debastiani, J. M. Dias, W. H. Liang, and E. Oset, Molecular $\Omega_{c}$ states within the local hidden gauge approach, arXiv: 1710.04231 .
[31] L. L. Chau, Quark Mixing in weak interactions, Phys. Rep. 95, 1 (1983).

[32] J. J. Wu and B. S. Zou, Prediction of super-heavy $N^{*}$ and $\Lambda^{*}$ resonances with hidden beauty, Phys. Lett. B 709, 70 (2012).

[33] A. Ashtekar, Symmetry reduced loop quantum gravity: A bird's eye view, Int. J. Mod. Phys. D 25, 1642010 (2016).

[34] T. Gershon (private communications). 